\title{
Effects of $\alpha$-lipoic acid on neurovascular function in diabetic rats: interaction with essential fatty acids
}

\author{
N.E. Cameron ${ }^{1}$, M. A. Cotter ${ }^{1}$, D.H. Horrobin ${ }^{2}$, H.J. Tritschler ${ }^{3}$ \\ ${ }^{1}$ Department of Biomedical Sciences, University of Aberdeen, Scotland, UK \\ ${ }^{2}$ Scotia Pharmaceuticals, Stirling, Scotland, UK \\ ${ }^{3}$ ASTA Medica, Frankfurt am Main, Germany
}

\begin{abstract}
Summary Elevated oxidative stress and impaired $n-6$ essential fatty acid metabolism contribute to defective nerve conduction velocity ( $\mathrm{NCV}$ ) and perfusion in diabetic rats, which may be corrected by free radical scavenger and $\gamma$-linolenic acid (GLA) treatments. $\alpha$-Lipoic acid (LPA) has antioxidant actions and both LPA racemate (racLPA) and GLA treatments produced benefits in clinical neuropathy trials. The aims were to study LPA action on neurovascular function in diabetic rats and to investigate potential interactions for co-treatment with GLA and other essential fatty acids. After 6 weeks of diabetes, 2 weeks of racLPA treatment corrected $20 \%$ sciatic motor and $14 \%$ saphenous sensory NCV deficits. The $\mathrm{ED}_{50}$ for motor NCV restoration was approximately $38 \mathrm{mg}$ $\mathrm{kg}^{-1}$ day $^{-1}$. racLPA also corrected a $49 \%$ diabetic deficit in sciatic endoneurial blood flow. $\mathrm{R}$ and S-LPA enantiomers were equipotent in correcting NCV and blood flow deficits. Treatment of diabetic rats with low doses $\left(20 \mathrm{mg} \mathrm{kg}^{-1} \mathrm{day}^{-1}\right)$ of racLPA and GLA,
\end{abstract}

while having modest effects on their own, showed evidence of marked synergistic action in joint treatment, completely correcting motor NCV and blood flow deficits. This was also noted for the novel compound, SOC0150, which contains equimolar proportions of LPA and GLA (ED $50.3 \mathrm{mg} \mathrm{kg}^{-1}$ day $^{-1}$, containing $3.5 \mathrm{mg}$ LPA). NCV effects also showed marked synergism when racLPA:GLA ratios were varied over a 1:3-3:1 range. In contrast, a compound containing LPA and the $n-3$ component, docosahexaenoic acid, showed similar activity to LPA alone. Thus, LPA-GLA interactions yield drug combinations and compounds with an order of magnitude increase in efficacy against experimental diabetic neuropathy and are worthy of consideration for clinical trials. [Diabetologia (1998) 41: 390-399]

Keywords Neuropathy, nerve conduction, blood flow, essential fatty acid, $\gamma$-linolenic acid, docosahexaenoic acid, lipoic acid, antioxidant, diabetic rat.
Oxidative stress contributes to the vascular and neuropathic complications of experimental diabetes mellitus. This is evidenced by measures of tissue oxidant damage such as nerve lipid peroxidation and changes in antioxidant protection systems, for example de-

Received: 22 September 1997 and in revised form: 26 November 1997

Corresponding author: Dr. N. E. Cameron, Department of Biomedical Sciences, University of Aberdeen, Marischal College, Aberdeen AB9 1AS, Scotland, UK

Abbreviations: NCV, Nerve conduction velocity; LPA, $\alpha$-lipoic acid; racLPA, $\alpha$-lipoic acid racemate; GLA, $\gamma$-linolenic acid; DHA, docosahexaenoic acid; NO, nitric oxide; GSH, reduced form of glutathione. creased nerve reduced glutathione (GSH) and superoxide dismutase content $[1,2]$. Functional effects of antioxidant treatment include protection of vascular endothelium function [3-5], and improved nerve blood flow, conduction velocity (NCV) and regenerative capacity $[2,6-10]$. $\alpha$-Lipoic acid (LPA) is a naturally occurring free radical scavenger and transition metal chelator. LPA is also a cofactor for mitochondrial pyruvate dehydrogenase and has been termed a "metabolic antioxidant" [11]. Recent studies from Low's group $[2,12]$ showed that treatment with LPA racemate (racLPA) prevented the development of digital sensory NCV deficits, impaired sciatic nerve blood flow, diminished nerve GSH, and deficient glucose uptake by neural tissues in diabetic rats. Short- 
term clinical trials of symptomatic and cardiac autonomic neuropathy showed some beneficial effects of racLPA treatment $[13,14]$. R-LPA may be more effective than the $S$ enantiomer in increasing tissue glucose uptake and metabolism and also as an antioxidant $[15,16]$, although the latter has been disputed for nervous tissue in vitro [17]. One aim of the investigation was to further characterise LPA effects on NCV and endoneurial blood flow in diabetic rats, including a comparison of $\mathrm{R}$ and $\mathrm{S}$ enantiomers.

The $n$ - 6 essential fatty acid, $\gamma$-linolenic acid (GLA), prevents and corrects nerve conduction abnormalities in diabetic rats, primarily through improved production of cyclooxygenase-derived vasodilator prostanoids that increase vasa nervorum perfusion $[18,19]$. Beneficial GLA effects were found in clinical diabetic neuropathy trials [20]. Previous rat studies revealed synergistic interactions between treatments improving prostanoid synthesis and those correcting nerve function via the vasa nervorum nitric oxide (NO) system, such as aldose reductase inhibitors (ARIs) and antioxidants [21-23]. Thus, a second aim was to assess the effects of joint LPA-GLA treatments both as individual drugs and using a novel compound, SOC0150, GLA-LPA.

\section{Materials and methods}

Experiments were performed in accordance with regulations specified in the United Kingdom "Animal Procedures Act, 1986" and the National Institutes of Health "Principles of Laboratory Animal Care, 1985 revised version".

Experimental groups and diabetes induction. Male SpragueDawley rats (Aberdeen University colony) were used, aged 19 weeks at the start of experiments. Non-diabetic rats were studied at this age as onset controls, to provide a starting value against which to judge any diabetes induced neuropathic deterioration. In this mature rat model, there is no significant difference between NCV or blood flow values in onset and agematched control groups [24-26]. Diabetes was induced by streptozotocin (Zeneca, Macclesfield, Cheshire, UK) freshly made up in sterile $154 \mathrm{mmol} \mathrm{l}^{-1} \mathrm{NaCl}$ solution, at a dose of $40-45 \mathrm{mg} \mathrm{kg}^{-1}$ i. p. Diabetes was verified after $24 \mathrm{~h}$ by the presence of hyperglycaemia and glucosuria (Visidex II and Diastix; Ames, Slough, UK) in non-fasted rats. After final experiments, plasma glucose was estimated (GOD-Perid method; Boehringer Mannheim, Mannheim, Germany) on samples taken from a carotid artery cannula or the tail vein. Diabetes duration was 8 weeks and, where appropriate, treatment was given for the last 2 weeks.

There were two sets of investigations. In the first, the efficacy of LPA racemate ( $r a c$ LPA) for correcting proximal sciatic motor NCV deficits was estimated at doses of 20,50 and $100 \mathrm{mg} \mathrm{kg}^{-1}$ day $^{-1}$. For the highest dose, effects on sciatic endoneurial blood flow and saphenous sensory NCV were also measured. Finally, the effects of $\mathrm{R}$ and $\mathrm{S}$ enantiomers were compared at a dose of $40 \mathrm{mg} \mathrm{kg}^{-1}$ day $^{-1}$, which approximates the $\mathrm{EC}_{50}$ for $r a c$ LPA.

The second set of experiments examined the effects of low doses of racLPA (20 mg kg${ }^{-1}$ day $\left.^{-1}\right)$ and GLA ethyl monoester
(24.8 mg kg ${ }^{-1}$ day $^{-1}$, supplying $20 \mathrm{mg} \mathrm{kg}^{-1}$ day $^{-1}$ GLA), alone and as a joint treatment to examine potential synergistic interactions for blood flow and NCV. Further experiments studied the effects of SOC0150, 1-(z,z,Z-octadeca-6,9,12-trienoic acid)-3-(DL-1,2-dithiolane-3-pentanoic acid)-propyl diester, a novel compound containing equimolar amounts of GLA and LPA, to establish dose-response relations for NCV and to examine the effects of high dose treatment on endoneurial blood flow. Additional neurovascular measurements were made on a mixture of pure GLA and racLPA containing the equimolar proportions found in SOC0150. The effects of varying the LPA:GLA ratio on NCV were studied using racLPA and GLA monoester. Finally, to test whether essential fatty acid LPA synergistic interactions extended to the $n-3$ series, the effects of treatment with the novel compound, docosahexaenoic acid (DHA)-LPA were examined for NCV.

LPA (ASTA Medica, Frankfurt, Germany) powder was mixed with sterile saline and $\mathrm{NaOH}$ was added until the suspension dissolved. The $\mathrm{pH}$ was then brought to 7.4 with $\mathrm{HCl}$. The LPA solution was given by daily i.p. injection. For the interaction experiments, GLA, as the monoester or pure GLA (Scotia Pharmaceuticals, Stirling, UK) was given as a dietary supplement, dispersion of the appropriate dose of oil being aided by the addition of $9 \mathrm{ml}$ of sunflower oil in $1.5 \mathrm{~kg}$ of diet. Previous experiments have shown that this or higher levels of sunflower or corn oil (both contain $70 \%$ linoleic acid and were given at up to a $10 \%$ dietary supplement) treatment have no effect on NCV or blood flow in diabetic and nondiabetic rats $[24,26]$. For experiments on the effects of SOC0150 (Scotia Pharmaceuticals), which contains $38.5 \%$ w/w LPA and $49.8 \% \mathrm{w} / \mathrm{w}$ GLA, treatment was given as a dietary supplement with sunflower oil to aid dispersion as described above. In this case, comparison was made with a mixture of pure GLA and racLPA dispersed in sunflower oil and added to the diet. The DHA-LPA compound (Scotia Pharmaceuticals) was similarly dispersed in the diet with sunflower oil.

Nerve conduction velocity. At the end of the treatment period, rats were anaesthetised with thiobutabarbital (Zeneca) by intraperitoneal injection (50-100 $\mathrm{mg} \mathrm{kg}^{-1}$ ). The trachea was cannulated for artificial ventilation and a carotid cannula was used to monitor mean systemic blood pressure. Motor NCV was measured between sciatic notch and knee in the nerve branch to tibialis anterior muscle, which is representative of the whole sciatic nerve in terms of susceptibility to diabetes and treatment effects [24, 25]. Saphenous sensory NCV was measured between groin and ankle as previously described [25]. Rectal and nerve temperatures were monitored, and regulated between 36.5 and $37.5^{\circ} \mathrm{C}$.

Nerve blood flow. Sciatic endoneurial blood flow was measured by microelectrode polarography and hydrogen clearance as previously described $[6,21,26]$. Briefly, rats were given neuromuscular blockade using d-tubocurarine (Sigma, $2 \mathrm{mg} \cdot \mathrm{kg}^{-1}$ via the carotid cannula) and were artificially ventilated. The level of anaesthesia was monitored by observing any reaction of blood pressure to manipulation, and supplementary thiobutabarbitone was given as necessary. Body core temperature was maintained at $37-38^{\circ} \mathrm{C}$ and nerve temperature was controlled within the range $35-37^{\circ} \mathrm{C}$ by radiant heat applied to a pre-warmed mineral oil pool that bathed the exposed sciatic nerve. A glass-insulated $\mathrm{H}_{2}$-sensitive electrode was inserted into the middle of the nerve. $10 \% \mathrm{H}_{2}$ was added to the inspired gas, the proportions of $\mathrm{O}_{2}$ and $\mathrm{N}_{2}$ being adjusted to $20 \%$ and $70 \%$, respectively. When the electrode $\mathrm{H}_{2}$ current had stabilised, the $\mathrm{H}_{2}$ supply was shut off and the clearance monitored. This was repeated at another nerve site. Mono- or bi-exponen- 
Table 1. Body weight and plasma glucose values for groups in the $\alpha$-lipoic acid studies

\begin{tabular}{|c|c|c|c|c|}
\hline \multirow[t]{2}{*}{ Group } & \multirow[t]{2}{*}{$n$} & \multicolumn{2}{|c|}{ Body weight (g) } & \multirow[t]{2}{*}{ Plasma glucose $\left(\mathrm{mmol} \mathrm{l}^{-1}\right)$} \\
\hline & & Start & End & \\
\hline Non-diabetic control & 10 & $449 \pm 6$ & - & $5.9 \pm 0.3$ \\
\hline Diabetic control & 10 & $454 \pm 8$ & $320 \pm 12$ & $42.0 \pm 2.7$ \\
\hline Diabetic $+20 \mathrm{mg} \mathrm{kg}^{-1} \mathrm{day}^{-1}$ rac LPA & 8 & $460 \pm 4$ & $327 \pm 10$ & $42.8 \pm 1.4$ \\
\hline Diabetic + $50 \mathrm{mg} \mathrm{kg}^{-1} \mathrm{day}^{-1} \mathrm{rac}$ LPA & 7 & $464 \pm 5$ & $316 \pm 11$ & $45.4 \pm 1.9$ \\
\hline Diabetic + $100 \mathrm{mg} \mathrm{kg}^{-1}$ day $^{-1}$ rac LPA & 10 & $451 \pm 2$ & $340 \pm 8$ & $40.9 \pm 2.3$ \\
\hline Diabetic $+40 \mathrm{mg} \mathrm{kg}^{-1}$ day $^{-1} \mathrm{R}-\mathrm{LPA}$ & 14 & $469 \pm 7$ & $310 \pm 13$ & $40.9 \pm 1.2$ \\
\hline Diabetic $+40 \mathrm{mg} \mathrm{kg}^{-1}$ day $^{-1} \mathrm{~S}-\mathrm{LPA}$ & 12 & $474 \pm 5$ & $321 \pm 14$ & $41.5 \pm 1.8$ \\
\hline
\end{tabular}

Data are mean \pm SEM
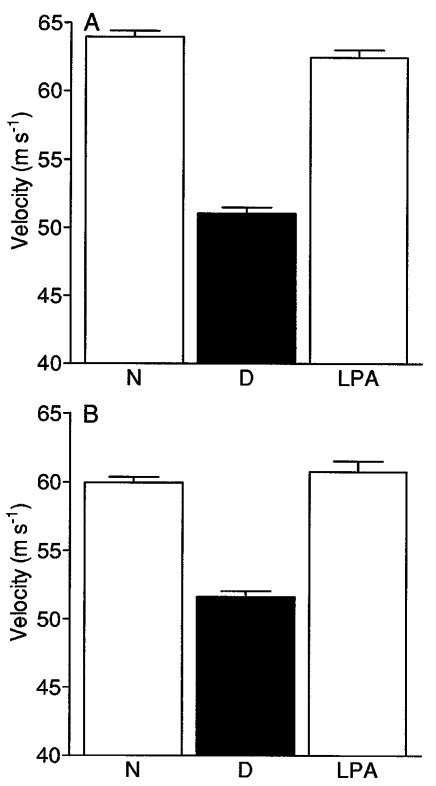

Fig.1A, B. Effects of diabetes and $\alpha$-lipoic acid racemate treatment on (A) sciatic motor conduction velocity and (B) saphenous sensory conduction velocity in rats. $\mathrm{N}$, nondiabetic group, $n=10 ; \mathrm{D}, 8$ week diabetic control group, $n=10$; LPA, 8 week diabetic group, treated for the last 2 weeks with $100 \mathrm{mg} \mathrm{kg}^{-1}$ day $^{-1} \alpha$-lipoic acid racemate, $n=10$. Data are mean \pm SEM. Statistics: motor conduction N or LPA vs D, $p<0.001 ; \mathrm{N}$ vs LPA, $p<0.05$. Sensory conduction $\mathrm{N}$ or LPA vs $\mathrm{D}, p<0.001$; $\mathrm{N}$ vs LPA, NS

tial curves were fitted to the data by regression analysis (Prism; Graphpad, San Diego, Calif., USA). The slow exponent was taken to reflect nutritive capillary flow [27]. Vascular conductance was calculated by dividing blood flow by mean arterial blood pressure. Endoneurial perfusion parameters were averaged from the two clearance measurements.

Statistical analysis. Data are expressed as mean \pm SEM. They were first subjected to Bartlett's test for homogeneity of variances and were given a log transformation if necessary (all blood flow and vascular conductance data for the interaction studies). One-way analysis of variance was then performed, followed by the Student-Newman-Keuls test to estimate the significance of differences for individual between-group comparisons. Where log transformation failed to equalize group variances (blood pressure, LPA study) the non-parametric Kruskal-Wallis analysis of variance was used, followed by Dunn's multiple comparison test. For the interaction study, dif- ferences between observed and predicted treatment effects were analysed using one sample Student's $t$-tests. A value of $p$ less than 0.05 was considered statistically significant. Sigmoid dose-response curves were constructed using the Marquardt algorithm and the least squares method for optimizing goodness-of-fit, using commercial software (Prism).

\section{Results}

Effects of $\alpha$-lipoic acid racemate treatment on nerve conduction velocity and sciatic endoneurial blood flow in diabetic rats. Data for body weights and plasma glucose concentration are given in Table 1. Eight weeks of diabetes caused an approximate $30 \%$ reduction in body weight and plasma glucose was elevated approximately 7 -fold. This was not affected by LPA treatment for the final 2 weeks.

$\mathrm{NCV}$ for sciatic motor fibres to tibialis anterior muscle (Fig.1A) and saphenous sensory fibres (Fig. 1B) were reduced by 8 weeks of diabetes to the extent of $20.2 \pm 0.7 \%(p<0.001)$ and $13.9 \pm 0.7 \%$ $(p<0.001)$, respectively. Treatment with $100 \mathrm{mg} \mathrm{kg}^{-1}$ day $^{-1}$ racLPA for the last 2 weeks corrected the motor $\mathrm{NCV}$ deficit by $88.2 \pm 4.4 \%(p<0.001)$ although the remaining small deficit was statistically significant $(p<0.05)$ compared to the non-diabetic group. Saphenous sensory NCV was completely corrected $(p<0.001)$ by racLPA.

Sciatic endoneurial blood flow (Fig.2A), $48.6 \pm 2.9 \%$ reduced $(p<0.001)$ by diabetes, was largely $(78.7 \pm 9.4 \% ; p<0.001)$ corrected by racLPA $\left(100 \mathrm{mg} \mathrm{kg}^{-1} \mathrm{day}^{-1}\right)$ treatment, the resultant value being within the non-diabetic range. Compared to the non-diabetic group, mean systemic blood pressure (Fig. 2B) tended to be reduced ( $\sim 18 \%)$ in both diabetic control $(p<0.05)$ and racLPA-treated groups $(p<0.01)$. Because vasa nervorum has poor or absent pressure autoregulation [27], such pressure variations would influence blood flow. To take pressure into account, the flow data are expressed as nutritive vascular conductance in Figure 2C. This was $41.4 \pm 3.6 \%$ $(p<0.001)$ reduced by diabetes, the deficit being completely corrected $(p<0.001)$ by racLPA treatment. 

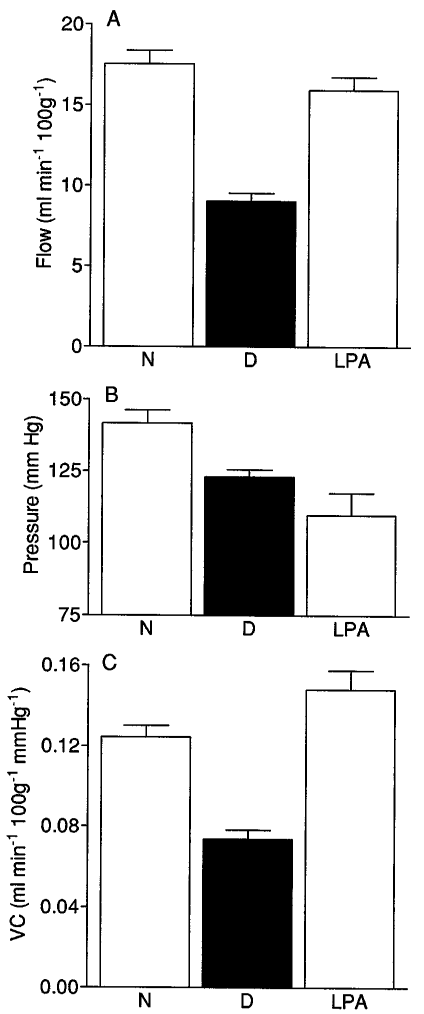

Fig. 2A-C. Effects of diabetes and $\alpha$-lipoic acid racemate treatment on (A) sciatic nutritive endoneurial blood flow, (B) mean systemic blood pressure and (C) endoneurial vascular conductance in rats. $\mathrm{N}$, non-diabetic group, $n=10 ; \mathrm{D}, 8$ week diabetic control group, $n=10$; LPA, 8 week diabetic group, treated for the last 2 weeks with $100 \mathrm{mg} \mathrm{kg}^{-1}$ day $^{-1} \alpha$-lipoic acid racemate, $n=8$. Data are mean \pm SEM. Statistics: blood flow and vascular conductance, $\mathrm{N}$ or LPA vs $\mathrm{D}, p<0.001 ; \mathrm{N}$ vs LPA, NS. Blood pressure, $\mathrm{N}$ vs $\mathrm{D}, p<0.05 ; \mathrm{N}$ vs LPA, $p<0.01$; D vs LPA, NS

Comparison of the effects of $R$ - and $S$ - enantiomers of $\alpha$-lipoic acid on motor nerve conduction velocity and sciatic endoneurial blood flow in diabetic rats. Motor NCVs for 3 doses $\left(20,50\right.$ and $100 \mathrm{mg} \mathrm{kg}^{-1}$ day $^{-1}$ ) of racLPA are shown in Figure 3, all of which caused a significant $(p<0.001)$ improvement compared to untreated diabetes. The $\operatorname{LogED}_{50}$ was $1.58 \pm 0.02$, which corresponds to an $\mathrm{ED}_{50}$ of approximately $38 \mathrm{mg} \mathrm{kg}^{-1}$ day $^{-1}$. Figure 3 also shows the effects of pure R-LPA or S-LPA enantiomers at a dose $\left(40 \mathrm{mg} \mathrm{kg}^{-1} \mathrm{day}^{-1}\right)$ chosen to approximate the middle of the dose-response curve for racLPA. Motor NCV was similar $\left(\sim 58.3 \mathrm{~m} \mathrm{~s}^{-1}\right)$ for both R- and S-LPA, significantly improved compared to untreated diabetes $(p<0.001)$ but reduced compared to the non-diabetic control group $(p<0.001)$. The degree of NCV correction was similar to expectations from the racLPA dose-response curve for both R- and S-LPA. Thus, observed values were not significantly different from the $57.8 \mathrm{~m} \mathrm{~s}^{-1}$ predicted by the curve for $40 \mathrm{mg} \mathrm{kg}^{-1}$ day $^{-1}$ racLPA, or from the observed NCV $\left(59.4 \pm 0.6 \mathrm{~m} \mathrm{~s}^{-1}\right)$ for the $50 \mathrm{mg} \mathrm{kg}{ }^{-1}$ day $^{-1}$ racLPA

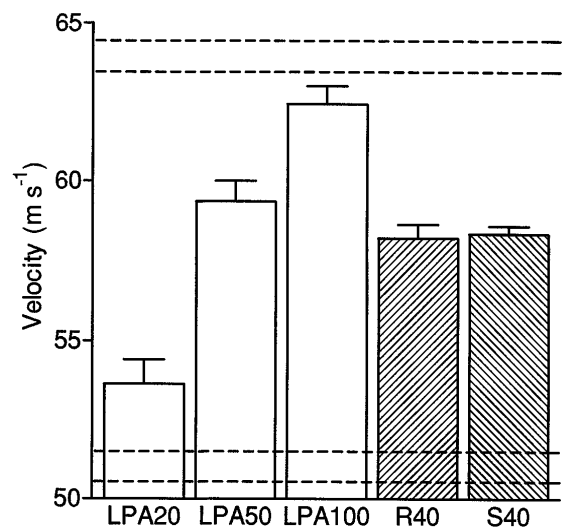

Fig. 3. Dose-response relationship for correction of sciatic motor conduction velocity by $\alpha$-lipoic acid racemate treatment and the effects of $\mathrm{R}$ and $\mathrm{S}$ enantiomers in diabetic rats. LPA20, 50 and 100, rats diabetic for 6 weeks then treated for 2 weeks with by $\alpha$-lipoic acid racemate at doses of 20,50 and $100 \mathrm{mg} \mathrm{kg}^{-1}$ day $^{-1}, n=7-10$; R40 and S40 6 week diabetic rats treated for a further 2 weeks with $\mathrm{R}$ or $\mathrm{S}$ enantiomers of $\alpha$-lipoic acid at a dose of $40 \mathrm{mg} \mathrm{kg}^{-1} \mathrm{day}^{-1}, n=10$. Data are mean \pm SEM. Upper and lower sets of dashed lines represent the envelope of mean \pm SEM of representative non-diabetic and diabetic control groups respectively, from data in Figure 1. Statistics: R40 vs S40, NS; R40 or S40 vs LPA50, NS; R40 or S40 vs LPA20 or LPA100, $p<0.001$; R40, S40, LPA20, LPA50 or LPA100 vs diabetic control group, $p<0.001$; R40, S40, LPA20 or LPA50 vs non-diabetic group, $p<0.001$; LPA100 vs non-diabetic group, $p<0.05$

group. Nutritive endoneurial blood flow was similar in R-LPA and S-LPA treated groups, being $12.45 \pm 0.49 \mathrm{ml} \mathrm{min}{ }^{-1} 100 \mathrm{~g}^{-1}$ and $12.06 \pm 0.83 \mathrm{ml}$ $\min ^{-1} 100 \mathrm{~g}^{-1}$, respectively. Corresponding values for mean systemic blood pressure were $102.4 \pm 4.5 \mathrm{~mm}$ $\mathrm{Hg}$ and $110.7 \pm 5.8 \mathrm{~mm} \mathrm{Hg}$. Neither of these parameters differed significantly between the two groups.

Interaction between $\alpha$-lipoic acid and $\gamma$-linolenic acid treatments, the effects of SOC0150, and a compound containing $\alpha$-lipoic acid and the n-3 component, docosahexaenoic acid. Eight weeks of diabetes caused hyperglycaemia and an approximate $25 \%$ reduction in body weight (Table 2), which were not affected by treatment during the final 2 weeks.

There was a $19.7 \pm 0.8 \%(p<0.001)$ deficit in sciatic motor NCV after 8 weeks of diabetes (Fig.4). This was partially corrected by low dose $\left(20 \mathrm{mg} \mathrm{kg}^{-1}\right.$ day $^{-1}$ i.p.) $r a c L P A ~(21.6 \pm 4.0 \% ; p<0.01)$ or low dose $\left(20 \mathrm{mg} \mathrm{kg} \mathrm{kg}^{-1}\right.$ day $^{-1}$ p.o.) GLA monoester $(18.7 \pm 3.8 \% ; p<0.01)$ treatments alone over the last 2 weeks. However, in diabetic rats given joint racLPA-GLA monoester treatment, the degree of NCV correction was $93.2 \pm 4.1 \%(p<0.001)$, the resultant value being in the non-diabetic range. The predicted NCV value for the joint treatment group was $55.7 \mathrm{~m} \mathrm{~s}^{-1}$ on the assumption of additivity of drug effects. The observed value of $62.9 \pm 0.5 \mathrm{~m} \mathrm{~s}^{-1}$ substantially exceeded this prediction $(p<0.0001)$, 
Table 2. Body weights and plasma glucose for rat groups used in the $\alpha$-lipoic acid: essential fatty acid interaction studies

\begin{tabular}{lrlrl}
\hline Group & $n$ & \multicolumn{2}{l}{ Weight $(\mathrm{g})$} & ${\text { Plasma glucose }\left(\mathrm{mmol}^{-1}\right)}$ \\
\cline { 3 - 4 } & & Start & End & $8.5 \pm 0.4$ \\
\hline Non-diabetic & 10 & $451 \pm 6$ & & $41.2 \pm 1.6$ \\
Diabetic & & $453 \pm 6$ & $333 \pm 14$ & $43.6 \pm 1.7$ \\
Untreated & 10 & $469 \pm 9$ & $343 \pm 6$ & $41.9 \pm 1.6$ \\
rac LPA & 8 & $472 \pm 6$ & $341 \pm 7$ & $39.2 \pm 1.8$ \\
GLA & 11 & $476 \pm 7$ & $352 \pm 11$ & $41.2 \pm 0.9$ \\
rac LPA + GLA & 12 & $457 \pm 3$ & $362 \pm 8$ & $43.2 \pm 1.8$ \\
SOC0150 & 25 & $456 \pm 3$ & $356 \pm 10$ & $40.1 \pm 1.9$ \\
rac LPA + GLA & 9 & $466 \pm 10$ & $365 \pm 16$ & \\
LPA-DHA & 8 & & & \\
\hline Data & & &
\end{tabular}

Data are mean \pm SEM

${ }^{a}$ Doses matched for comparison with SOC0150

indicating a synergistic interaction between the two treatments.

Figure 4 also shows the effects of dietary supplementation with a dose of $48 \mathrm{mg} \mathrm{kg}^{-1}$ day $^{-1}$ SOC0150, (which would give doses of $23.4 \mathrm{mg} \mathrm{kg}^{-1}$ day $^{-1}$ GLA and $18.1 \mathrm{mg} \mathrm{kg}^{-1} \mathrm{day}^{-1}$ LPA on complete hydrolysis of the ester), and joint treatment with the same amounts of pure GLA and racLPA by dietary supplementation. These treatments corrected NCV by $98.7 \pm 6.4 \%(p<0.001)$ and $93.1 \pm 6.0 \%(p<0.001)$, respectively; the resultant values were not significantly different from each other or from the NCV value for the non-diabetic group. Based on a consideration of the NCV dose-response curves for GLA [19, 23] and racLPA, the predicted NCV value for additive effects between the two components was $56.0 \mathrm{~m} \mathrm{~s}^{-1}$. This was substantially exceeded with SOC0150 $(p<0.0001)$ and the corresponding GLA-racLPA treatments $(p<0.0001)$ indicating synergistic interactions. NCV was also examined in two further groups $(n=6-8)$ of SOC0150 treated diabetic rats at doses of 5 and $16 \mathrm{mg} \mathrm{kg}^{-1} \mathrm{day}^{-1}$ to enable the dose-response curve to be estimated. This is shown in Figure 4B, in terms of LPA content, for comparison with the racLPA dose-response relationship. The $\log \mathrm{EC}_{50}$ for SOC0150 was $0.968 \pm 0.046$, corresponding to a dose of approximately $9.3 \mathrm{mg} \mathrm{kg} \mathrm{kg}^{-1} \mathrm{day}^{-1}$ (which would give $\sim 3.5 \mathrm{mg} \mathrm{kg}^{-1} \mathrm{day}^{-1} \mathrm{LPA}$ and $\sim 4.5 \mathrm{mg} \mathrm{kg}^{-1} \mathrm{day}^{-1}$ GLA). Figure 4B also shows data for a group of diabetic rats $(n=8)$ treated with DHA-LPA, at a dose of $50.4 \mathrm{mg} \mathrm{kg}^{-1}$ day $^{-1}$ (which, when hydrolysed would yield $18.1 \mathrm{mg} \mathrm{kg}^{-1}$ day $^{-1} \mathrm{LPA}$ and $27.4 \mathrm{mg} \mathrm{kg}^{-1}$ day $^{-1}$ DHA). NCV was $54.0 \pm 0.4 \mathrm{~m} \mathrm{~s}^{-1}$, which did not differ significantly from the $53.3 \mathrm{~m} \mathrm{~s}^{-1}$ predicted from the racLPA dose-response curve for the LPA component alone.

Sciatic nutritive endoneurial blood flow (Fig. 5A), $48.4 \pm 2.4 \%$ reduced by 8 weeks of diabetes, was not significantly affected by racLPA $(41.8 \pm 5.1 \%$ deficit $)$ or GLA $(41.7 \pm 2.7 \%$ deficit $)$ treatment alone. However, for joint racLPA-GLA treatment, flow was in the non-diabetic range, significantly elevated com- pared to diabetic control and single treatment groups $(p<0.001)$. This was also noted for the SOC0150treated group and rats treated jointly with comparable doses of pure GLA and racLPA.

Compared to the non-diabetic group, mean systemic blood pressure (Fig. 5B) tended to be reduced $(\sim 14 \%)$ by diabetes regardless of treatment and this was statistically significant for diabetic control, racLPA and GLA-treated groups $(p<0.05)$. Nutritive endoneurial vascular conductance (Fig. 5C) was $39.6 \pm 2.2 \%(p<0.001)$ reduced by diabetes, not significantly altered by racLPA or GLA treatments alone, but completely corrected by joint racLPAGLA treatment $(p<0.001)$. A similar correction of vascular conductance was observed using SOC0150, and pure GLA with racLPA cotreatment (both $p<0.001$ vs diabetic control group, NS vs the non-diabetic group). For nutritive blood flow and vascular conductance, the magnitude of the joint GLAracLPA treatment increment exceeded additivity predictions by 3.7-fold $(p=0.004)$ and 3.3-fold $(p=0.012)$, respectively, indicating a synergistic interaction for nutritive perfusion.

NCV results from an experiment examining lower dose GLA - racLPA mixtures and ratiometric effects are shown in Table 3. A mixture of $6.7 \mathrm{mg} \mathrm{kg}^{-1}$ day $^{-1}$ racLPA given by daily i.p. injection and $6.7 \mathrm{mg} \mathrm{kg}^{-1}$ day $^{-1}$ GLA as the monoester, gave an NCV that far exceeded the expected value for additivity. Based on dose-response curves for GLA and racLPA, the excess NCV above the expected value could be produced by an average 6-fold increase in GLA or racLPA dose. Treatment with racLPA - GLA monoester mixtures in 3:1 and 1:3 ratios were also examined (Table 3 ). In both cases, whether racLPA or GLA was predominant, observed motor NCV exceeded additive predictions, indicating a substantial synergy between the treatments. When racLPA was in relative excess, the synergistic effect was equivalent to an average 3.8-fold increase in racLPA or GLA dose whereas when GLA was predominant the equivalence was for a 5.1-fold increased dose. 

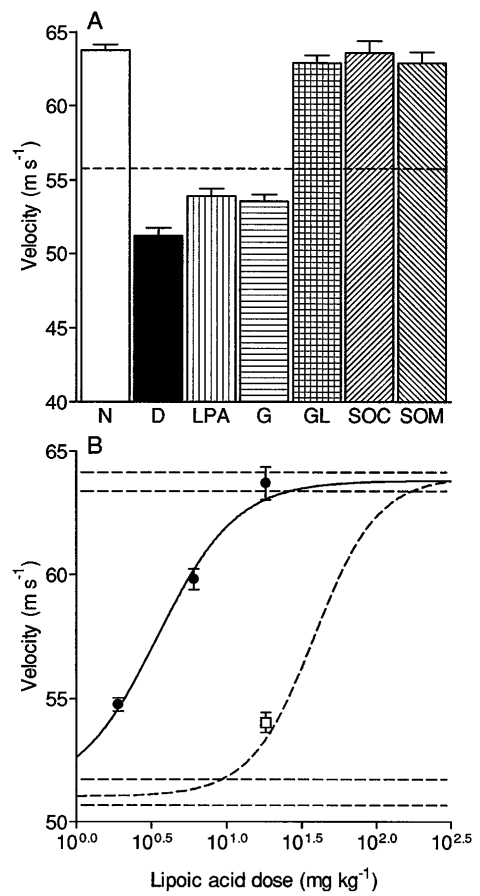

Fig. 4A, B. Effects of diabetes and treatments containing low doses of $\alpha$-lipoic acid and $\gamma$-linolenic acid, alone and in combination $(\mathbf{A})$, on sciatic motor conduction velocity and $(\mathbf{B})$ the dose response relationship for SOC0150 and effects of docosahexaenoic acid lipoic acid. $\mathrm{N}$, non-diabetic group, $n=10 ; \mathrm{D}, 8$ week diabetic control group, $n=10$. Other groups were diabetic for 8 weeks and treated for the last 2 weeks with either $20 \mathrm{mg} \mathrm{kg}^{-1}$ day $^{-1} \alpha$-lipoic acid racemate (LPA, $n=8$ ), $20 \mathrm{mg}$ $\mathrm{kg}^{-1}$ day $^{-1} \gamma$-linolenic acid (G, $\left.n=11\right), 20 \mathrm{mg} \mathrm{kg}^{-1}$ each of $\alpha$-lipoic acid racemate and $\gamma$-linolenic acid (GL, $n=12), 48 \mathrm{mg}$ $\mathrm{kg}^{-1}$ day $^{-1} \mathrm{SOC} 0150$ (SOC, $n=10$ ), or a combination of $18.1 \mathrm{mg} \mathrm{kg}^{-1}$ day $^{-1} \alpha$-lipoic acid racemate and $23.4 \mathrm{mg} \mathrm{kg}^{-1}$ day $^{-1} \gamma$-linolenic acid to mimic SOC0150 treatment (SOM, $n=9)$. The horizontal dashed line in A shows the expected conduction velocity level for combined treatment in the GL group, calculated from LPA and G group data assuming additivity of effects. B shows the dose response relationship for SOC0150 (O, $n=7-10$ ) and the best fit sigmoid (solid curve). The dose response relationship for $\alpha$-lipoic acid racemate is given for comparison (dashed curve). Conduction velocity data for docosahexaenoic acid - lipoic acid $(\square, n=8)$ at a daily dose of $50.4 \mathrm{mg} \mathrm{kg}^{-1} \mathrm{day}^{-1}$. Upper and lower sets of dashed lines represent the envelope of mean \pm SEM of representative non-diabetic and diabetic control groups respectively, from data in Figure 4A. Data are mean \pm SEM. Statistical analysis: motor conduction velocity, D vs LPA and $\mathrm{G}, p<0.01$; $\mathrm{D}$ vs all other groups, $p<0.001$; G or LPA vs N, GL, SOC or SOM, $p<0.001$; all other comparisons NS

\section{Discussion}

The data show that racLPA is effective in correcting $\mathrm{NCV}$ and nerve perfusion abnormalities in diabetic rats. This provides additional information to complement a previous racLPA study by Nagamatsu et al. [2], which used a preventive design over 1-3 months of diabetes. However, there are some differences in the NCV findings. While that study showed dose-de-
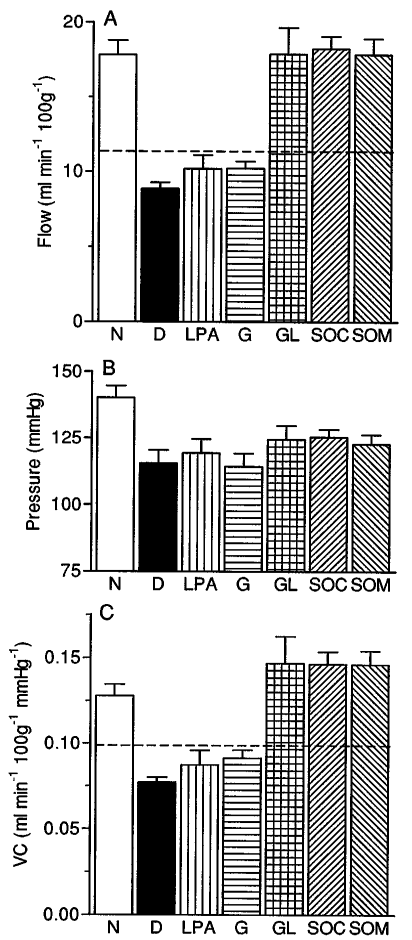

Fig. 5A-C. Effects of diabetes and treatments containing low doses of $\alpha$-lipoic acid and $\gamma$-linolenic acid, alone and in combination on (A) sciatic nutritive endoneurial blood flow, (B) mean systemic blood pressure and $(\mathbf{C})$ endoneurial vascular conductance (VC). $\mathrm{N}$, non-diabetic group, $n=10$; D, 8 week diabetic control group, $n=10$. Other groups were diabetic for 8 weeks and treated for the last 2 weeks with either $20 \mathrm{mg}$ $\mathrm{kg}^{-1}$ day $^{-1} \alpha$-lipoic acid racemate (LPA, $n=8$ ), $20 \mathrm{mg} \mathrm{kg}^{-1}$ day $^{-1} \gamma$-linolenic acid (G, $\left.n=11\right), 20 \mathrm{mg} \mathrm{kg}^{-1}$ day $^{-1}$ each of $\alpha$-lipoic acid racemate and $\gamma$-linolenic acid (GL, $n=11$ ), $48 \mathrm{mg}$ $\mathrm{kg}^{-1}$ day $^{-1} \mathrm{SOC} 0150$ (SOC, $n=10$ ), or a combination of $18.1 \mathrm{mg} \mathrm{kg}^{-1}$ day $^{-1} \alpha$-lipoic acid racemate and $23.4 \mathrm{mg} \mathrm{kg}^{-1}$ day $^{-1} \gamma$-linolenic acid to mimic SOC0150 treatment (SOM, $n=9)$. Data are mean + SEM. The horizontal dashed lines in $\mathbf{A}$ and $\mathbf{C}$ shows the expected flow and conductance levels for combined treatment in the GL group, calculated from LPA and $G$ group data assuming additivity of effects. Statistical analysis: blood flow and vascular conductance, D, LPA or G vs N, GL, SOC or SOM, $p>0.001$; all other comparisons NS. Blood pressure, $\mathrm{N}$ vs D or $\mathrm{G}, p<0.01$; $\mathrm{N}$ vs LPA, $p<0.05$; all other comparisons NS

pendent protection of hind limb digital sensory $\mathrm{NCV}$, motor NCV in sciatic tibial-interosseous and caudal nerves was not significantly altered. However, in their model, diabetes was induced in young (8 weeks of age) rats and part of the NCV deficit depends on blunting of the axial growth and maturation of nerve fibres $[28,29]$ so that motor NCV deficits are relative to age-matched rather than onset control values. In contrast, in our mature rat model using proximal sciatic nerve fibres, motor NCV changes are relative to onset controls and there is little further maturation effect $[24,25]$. The sciatic tibial-interosseous preparation is also more resistant to diabetes than the proximal sciatic branches [24, 30], and may be 
Table 3. Body weights, plasma glucose, conduction velocity values and degree of drug amplification effects for $\gamma$-linolenic: $\alpha$-lipoic acid mixtures

\begin{tabular}{|c|c|c|c|c|c|c|c|}
\hline \multirow[t]{2}{*}{ Group } & \multirow[t]{2}{*}{$n$} & \multicolumn{2}{|c|}{ Body weight (g) } & \multirow{2}{*}{$\begin{array}{l}\text { Plasma glucose } \\
\left(\mathrm{mmol} \mathrm{l}^{-1}\right)\end{array}$} & \multicolumn{2}{|c|}{ Motor conduction velocity (NCV) } & \multirow{2}{*}{$\begin{array}{l}\text { Average increase in } \\
\text { GLA/LPA dose } \\
\text { required to match } \\
\text { observed NCV }\end{array}$} \\
\hline & & Start & End & & $\begin{array}{l}\text { Observed } \\
\left(\mathrm{m} \mathrm{s}^{-1}\right)\end{array}$ & $\begin{array}{l}\text { Expected } \\
\left(\mathrm{m} \mathrm{s}^{-1}\right)\end{array}$ & \\
\hline GLA 6.7 rac LPA 6.7 & 8 & $454 \pm 5$ & $347 \pm 6$ & $38.8 \pm 0.9$ & $58.73 \pm 0.53^{\mathrm{a}}$ & 52.62 & 6.0-fold \\
\hline GLA 6.7 rac LPA 20 & 8 & $458 \pm 5$ & $329 \pm 11$ & $43.1 \pm 1.9$ & $60.14 \pm 0.49^{\mathrm{a}}$ & 54.87 & 3.7-fold \\
\hline GLA 20 rac LPA 6.7 & 8 & $454 \pm 4$ & $329 \pm 11$ & $40.0 \pm 1.7$ & $60.15 \pm 0.44^{\mathrm{a}}$ & 53.84 & 5.1-fold \\
\hline
\end{tabular}

Data are mean \pm SEM; drug doses are in $\mathrm{mg} \mathrm{kg}^{-1}$ day $^{-1}$.

a Significantly different from expected NCV, $p<0.001$, one sample Student's $t$-test

less amenable to some treatments such as aldose reductase inhibitors [25]. A similar discrepancy between maturation effects and different nerve branches was noted for aminoguanidine treatment. Thus, effects on sciatic tibial-interosseous nerve in young diabetic rats were delayed until 4 months of aminoguanidine treatment [31]. However, in proximal sciatic motor nerves, aminoguanidine prevented and rapidly reversed NCV deficits in mature diabetic rats [32]. Thus, it is plausible that discrepant observations of racLPA effects on motor $\mathrm{NCV}$ reflect the nerve branch studied, the duration of treatment and the age at which diabetes was induced. Nonetheless, the data clearly show that racLPA corrects motor and sensory NCV defects, as do several other antioxidants including vitamin $\mathrm{E}$, probucol and acetyl cysteine $[6,7,9,10]$.

racLPA corrected sciatic endoneurial blood flow deficits in a dose-dependent manner, $100 \mathrm{mg} \mathrm{kg}^{-1}$ day $^{-1}$ causing complete normalisation whereas $20 \mathrm{mg}$ $\mathrm{kg}^{-1}$ day $^{-1}$ had a minimal effect. This is in good agreement with the relationship described by Nagamatsu et al. [2]. In that preventive study, racLPA did not alter blood flow and NCV in non-diabetic rats, which concurs with data for other antioxidants $[6,8,9]$. Rand S-LPA enantiomers were equipotent in correcting NCV and blood flow deficits, the results fitting well with predictions from the dose-response curve for racLPA. R-LPA has been reported to be much more effective than S-LPA in increasing tissue glucose uptake and metabolism [15]. In diabetic rat sciatic nerve, racLPA treatment increased glucose uptake to compensate for a deficit in this parameter [12], presumably due mainly to the R-LPA component. The similarity of R-LPA and S-LPA actions in this investigation suggests that beneficial effects on nerve function do not depend upon promotion of glucose uptake under the conditions of these experiments; nerve glucose is already approximately 10fold elevated in our hyperglycaemic rat model [33]. However, under hypoglycaemic conditions, for example with intensive insulin treatment, LPA-dependent improvements in nerve glucose homeostasis might prevent the fibre degeneration characteristic of that state [34]. Arguably, a potential disadvantage of in- creased glucose uptake, coupled with hyperglycaemia, would be a further elevation in sciatic nerve polyol levels. Although not measured in this study, it was previously found that $100 \mathrm{mg} \mathrm{kg}^{-1}$ day $^{-1}$ racLPA treatment doubled nerve sorbitol content in diabetic rats [12]. However, the 3-11-fold sorbitol increases in diabetic and nondiabetic rats treated with a sorbitol dehydrogenase inhibitor did not have any adverse effects on NCV [33], suggesting that polyol accumulation is relatively unimportant.

LPA scavenges hydroxyl radicals, hyperchlorous acid, hydrogen peroxide, singlet oxygen and peroxynitrite, but not superoxide radicals. LPA also chelates transition metals, thus reducing free radical formation by glucose autoxidation and hydroxyl radical production by the Fenton reaction. Tissues can convert LPA to dihydro-LPA, which, although ineffective against singlet oxygen, has a broader spectrum of action than LPA, additionally scavenging superoxide and peroxyl radicals [11]. R- and S-LPA enantiomers are converted to dihydro-LPA by different routes; R-LPA is metabolised by dihydrolipoamide dehydrogenase in mitochondria, which requires NADH as cofactor. S-LPA is converted in the cytosol and mitochondrial matrix by glutathione reductase, which requires NADPH [11]. The LPA-dihydroLPA cycle may recycle major antioxidants including glutathione, ascorbate, thioredoxin and ubiquinone. In turn, these participate in vitamin $E$ regeneration from its oxidised radical [11], although this LPA effect has been disputed in vivo under conditions of vitamin E deficiency [35].

Conversion to dihydro-LPA may be important for LPA action because neutralisation of the endothelial vasodilator, NO, by superoxide contributes to reduced vasa nervorum blood flow in diabetic rats $[4$, 6, 21, 36-38]. Given that R and S-LPA are metabolised to dihydro-LPA by different pathways, the potential effect of diabetes requires consideration. Nerve mitochondrial NADH/NAD ${ }^{+}$ratio is increased by diabetes [39], as expected for endoneurial hypoxia $[27,40]$. This would favour the conversion of R-LPA to dihydro-LPA. However, the improvement in vasa nervorum blood flow by LPA would restore mitochondrial $\mathrm{NADH} / \mathrm{NAD}^{+}$, as noted for vasodilator 
treatment $[39,40]$, thereby negating this advantage. Synthesis of sorbitol from glucose by aldose reductase limits NADPH availability for glutathione reductase, which causes an ARI-correctable reduction in nerve GSH content in diabetes [41]. An NADPH deficit would impair dihydro-LPA production from S-LPA. Against that, however, the direct effect of LPA in recycling oxidised glutathione would bypass glutathione reductase; the diminished nerve GSH content in diabetic rats is prevented by racLPA treatment [2]. Thus, more enzyme and NADPH would be available for S-LPA to dihydro-LPA conversion. A "NADPH-sparing" effect of LPA could also benefit vasa nervorum endothelial function because NAD$\mathrm{PH}$ is a cofactor for NO synthase. racLPA treatment protects against defective corpus cavernosum NOmediated relaxation involving both vascular endothelium and nitrergic nerves in diabetic rats [42]. Thus, LPA effects are likely to be more complex than anticipated for a simple free radical scavenger, and beneficial actions of both R- and S-LPA on diabetic nerve function would be expected, in line with the observations.

The synergistic interaction between LPA and GLA in correcting NCV and blood flow deficits, whether given separately or combined as a novel compound, SOC0150, concurs with predictions from previous work. Thus, combined cyclooxygenase and NO synthase inhibitor treatment produced NCV defects in non-diabetic rats similar to those found in diabetes, and there was a substantial synergy between those drugs [43]. The interpretation was that cyclooxygenase inhibition reduced the synthesis of prostacyclin whereas NO synthase inhibition impaired vasodilation by NO, joint treatment disrupting the normal compensatory interactions between these complimentary endothelial vasodilator systems. The consequences of the diabetic deficit in the vasa nervorum NO system $[36,37]$ are greatly exacerbated by impaired prostacyclin synthesis [44], primarily due to reduced availability of arachidonic acid consequent to defective $\Delta-6$ desaturation of linoleic acid. This may be restored by GLA or arachidonic acid treatment $[19,40,45]$. Thus, joint treatment of diabetic rats with drugs targeting the prostacyclin (GLA) and vascular NO system (antioxidants and ARIs) [3-5, 40, $41,46]$ might be expected to show mutual facilitation. This was seen for nerve blood flow and NCV using a combination of GLA-rich evening primrose oil and aldose reductase inhibitors [21], GLA and a probucol analogue [22] and the novel drug, ascorbyl-GLA [23]. Because of LPA's neurovascular and NO system actions [2, 42] it was considered a good candidate for investigating potential interactions with GLA treatment. The degree of synergism between LPA and GLA was high, resulting in a drug mixture or the compound SOC0150 that had a several-fold increase in efficacy for correcting motor NCV and endoneurial blood flow defects. Experiments using different molar ratios of LPA and GLA showed that a synergistic interaction was present over a wide range of relative concentrations from 3:1 in favour of LPA to 3:1 in favour of GLA. However, the interaction was greatest when ratios were roughly equal or with GLA somewhat in excess. Thus, the 1.3:1 GLA:LPA ratio for SOC0150 is likely to be reasonably optimal.

Comparison of the effects of SOC0150 with that of DHA-LPA at a dose that contained the same LPA content, showed that DHA-LPA had a much lesser effect, not significantly better than that expected for LPA alone. DHA (22:6n-3) is an $n$-3 essential fatty acid metabolite, one elongation and desaturation step downstream of eicosapentaenoic acid 20:5n-3 (which is the $n-3$ homologue of arachidonic acid $20: 4 n-6)$. DHA is found in high concentrations at synaptic junctions and in the brain and is essential for the development and function of retina and brain [47]. DHA has vascular actions, including inhibition of cyclooxygenase-mediated prostanoid production from arachidonic acid which would reduce nerve blood flow; however, lipoxygenase products of DHA inhibit thromboxane $\mathrm{A}_{2}$ effects on platelets and vascular smooth muscle, which would tend to improve perfusion $[40,45,48]$. Thus, although DHA is important for structure and function of neural membranes, vascular effects are likely to be relatively neutral. This would explain the lack of interaction with LPA for NCV and contrasts with LPA-GLA mixtures and SOC0150 where GLA, via conversion to arachidonic acid, is a precursor of vasodilator prostanoids such as prostacyclin that increase nerve perfusion [40].

In conclusion, the data show an enantiomer-independent beneficial action of LPA on blood flow and $\mathrm{NCV}$ in diabetic rats. Furthermore, facilitatory interactions with joint GLA treatment greatly amplified these advantageous effects. Given that LPA and GLA both showed some benefits in clinical trials $[13,20]$, joint or SOC0150 treatment would be a logical elaboration offering enhanced efficacy.

Acknowledgements. This work was supported in part by a grant from the British Diabetic Association, and funds from Scotia Pharmaceuticals and ASTA Medica.

\section{References}

1. Low PA, Nickander KK (1991) Oxygen free radical effects in sciatic nerve in experimental diabetes. Diabetes 40: 873-877

2. Nagamatsu M, Nickander KK, Schmelzer JD et al. (1995) Lipoic acid improves nerve blood flow, reduces oxidative stress and improves distal nerve conduction in experimental diabetic neuropathy. Diabetes Care 18: 1160-1167

3. Keegan A, Walbank H, Cotter MA, Cameron NE (1995) Chronic vitamin E treatment prevents defective endothelium-dependent relaxation in diabetic rat aorta. Diabetologia 38: 1475-1478 
4. Archibald V, Cotter MA, Keegan A, Cameron NE (1996) Contraction and relaxation of aortas from diabetic rats: effects of chronic anti-oxidant and aminoguanidine treatments. Naunyn-Schmeideberg's Arch Pharmacol 353: 584-591

5. Rösen P, Ballhausen T, Bloch W, Addicks K (1995) Endothelial relaxation is disturbed by oxidative stress in the diabetic rat heart: influence of tocopherol as antioxidant. Diabetologia 38: 1157-1168

6. Cameron NE, Cotter MA, Archibald V, Dines KC, Maxfield EK (1994) Anti-oxidant and pro-oxidant effects on nerve conduction velocity, endoneurial blood flow and oxygen tension in non-diabetic and streptozotocin-diabetic rats. Diabetologia 37: 449-459

7. Cotter MA, Love A, Watt MJ, Cameron NE, Dines KC (1995) Effects of natural free radical scavengers on peripheral nerve and neurovascular function in diabetic rats. Diabetologia 38: 1285-1294

8. Cameron NE, Cotter MA (1995) Neurovascular deficits in diabetic rats: potential contribution of autoxidation and free radicals examined using transition metal chelating agents. J Clin Invest 96: 1159-1163

9. Love A, Cotter MA, Cameron NE (1996) Effects of the sulphydryl donor $N$-acetyl-L-cysteine on nerve conduction, perfusion, maturation and regeneration following freeze damage in diabetic rats. Eur J Clin Invest 26: 698-706

10. Love A, Cotter MA, Cameron NE (1996) Nerve function and regeneration in diabetic and galactosaemic rats: antioxidant and metal chelator effects. Eur J Pharmacol 314: 33-39

11. Packer L, Tritschler HJ, Wessel K (1996) Neuroprotection by the metabolic antioxidant $\alpha$-lipoic acid. Free Rad Biol Med 22: 359-378

12. Low PA, Schmelzer JD, Zollman PJ, Tritschler HJ (1996) $\alpha$-Lipoic acid prevents the deficit in glucose uptake in experimental diabetic neuropathy. Diabetologia 39 [Suppl 1]: A34 (Abstract)

13. Ziegler D, Hanefeld M, Ruhnau KJ et al. (1995) Treatment of symptomatic diabetic peripheral neuropathy with the anti-oxidant $\alpha$-lipoic acid. A 3-week multicentre randomized controlled trial (ALADIN Study). Diabetologia 38: $1425-1433$

14. Ziegler D, Schatz H, Gries FA, Ulrich H, Reichel G (1997) Effects of treatment with the antioxidant $\alpha$-lipoic acid on cardiac autonomic neuropathy in NIDDM patients. A 4month randomized controlled multicenter trial (DEKAN Study). Diabetes Care 20: 369-373

15. Estrada DE, Ewart HS, Tsakiridis T et al. (1996) Stimulation of glucose uptake by the natural coenzyme $\alpha$-lipoic acid / thioctic acid. Diabetes 45: 1798-1804

16. Maitra I, Serbinova E, Tritschler HJ, Packer L (1996) Stereospecific effects of R-lipoic acid on buthionine sulfoximine-induced cataract formation in newborn rats. Biochem Biophys Res Comm 221: 422-429

17. Nickander KK, McPhee BR, Low PA, Tritschler H (1996) Alpha-lipoic acid: antioxidant potency against lipid peroxidation of neural tissues in vitro and implications for diabetic neuropathy. Free Rad Biol Med 21: 631-639

18. Cameron NE, Cotter MA, Robertson S (1991) Effects of essential fatty acid dietary supplementation on peripheral nerve and skeletal muscle function and capillarization in streptozocin diabetic rats. Diabetes 40: 532-539

19. Dines KC, Cotter MA, Cameron NE (1995) Comparison of the effects of evening primrose oil and triglycerides containing $\gamma$-linolenic acid on nerve conduction and blood flow in diabetic rats. J Pharmacol Exp Ther 273: $49-55$
20. Keen H, Payan J, Allawi J et al. (1993) Treatment of diabetic neuropathy with $\gamma$-linolenic acid. Diabetes Care 16: 8-15

21. Cameron NE, Cotter MA, Hohman TC (1996) Interactions between essential fatty acid, prostanoid, polyol pathway and nitric oxide mechanisms in the neurovascular deficit of diabetic rats. Diabetologia 39: 172-182

22. Cameron NE, Cotter MA (1996) Interaction between oxidative stress and $\gamma$-linolenic acid in impaired neurovascular function of diabetic rats. Am J Physiol 261:E471-E476

23. Cameron NE, Cotter MA (1996) Comparison of the effects of ascorbyl $\gamma$-linolenic acid and $\gamma$-linolenic acid in the correction of neurovascular deficits in diabetic rats. Diabetologia 39: 1047-1054

24. Cameron NE, Cotter MA, Harrison J (1986) The effects of diabetes on motor nerve conduction velocity in different branches of the rat sciatic nerve. Exp Neurol 92: 757-761

25. Cameron NE, Cotter MA, Robertson S (1989) The effect of aldose reductase inhibition on the pattern of nerve conduction deficits in diabetic rats. Q J Exp Physiol 74: 917-926

26. Cameron NE, Cotter MA, Low PA (1991) Nerve blood flow in early experimental diabetes in rats: relation to conduction deficits. Am J Physiol 261:E1-E8

27. Low PA, Lagerlund TD, McManis PG (1989) Nerve blood flow and oxygen delivery in normal, diabetic and ischemic neuropathy. Int Rev Neurobiol 31: 355-438

28. Sharma AK, Bajada S, Thomas PK (1981) Influence of streptozotocin-induced diabetes on myelinated nerve fibre maturation and integrity and on body growth in the rat. Acta Neuropathol 53: 257-265

29. Malone JI, Lowitt S, Korthals JK, Salem A, Miranda C (1996) The effect of hyperglycemia on nerve conduction and structure is age dependent. Diabetes 45: 209-215

30. Greene DA, De Jesus PV Jr, Winegrad AI (1975) Effects of insulin and dietary myo-inositol on impaired peripheral motor nerve conduction velocity in acute streptozotocin diabetes. J Clin Invest 55: 1326-1336

31. Kihara M, Schmelzer JD, Poduslo JF, Curran FF, Nickander KK, Low PA (1991) Aminoguanidine effect on nerve blood flow, vascular permeability, electrophysiology, and oxygen free radicals. Proc Natl Acad Sci USA 88: 6107-6111

32. Cameron NE, Cotter MA (1996) Rapid reversal by aminoguanidine of the neurovascular effects of diabetes in rats: modulation by nitric oxide synthase inhibition. Metabolism 45: 1147-1152

33. Cameron NE, Cotter MA, Basso M, Hohman TC (1997) Comparison of the effects of inhibitors of aldose reductase and sorbitol dehydrogenase on neurovascular function, nerve conduction and tissue polyol pathway metabolites in streptozotocin-diabetic rats. Diabetologia 40: 271-281

34. Westfall SG, Felten DL, Mandelbaum JA, Moore SQ, Petersen RG (1983) Degenerative neuropathy in insulintreated diabetic rats. J Neurol Sci 61: 93-107

35. Podda M, Tritschler HJ, Ulrich H, Packer L (1994) Alphalipoic acid supplementation prevents symptoms of vitamin E deficiency. Biochem Biophys Res Commun 204: 98-104

36. Kihara M, Low PA (1995) Impaired vasoreactivity to nitric oxide in experimental diabetic neuropathy. Exp Neurol 132: $180-185$

37. Maxfield EK, Cameron NE, Cotter MA (1997) Effect of diabetes on reactivity of sciatic vasa nervorum in rats. J Diabet Complications 11: 47-55

38. Gryglewski RJ, Palmer RMJ, Moncado S (1986) Superoxide anion is involved in the breakdown of endothelium-derived vascular relaxing factor. Nature 320: 454-456 
39. Obrosova I, Van Heyningen D, Cao X, Stevens M, Greene D (1997) Metabolic compensations for diabetes-induced endoneurial hypoxia. J Peripher Nerv Syst 2: 290 (Abstract)

40. Cameron NE, Cotter MA (1994) The relationship of vascular changes to metabolic factors in diabetes mellitus and their role in the development of peripheral nerve complications. Diabetes Metab Rev 10: 189-224

41. Hohman TC, Banas D, Basso M, Cotter MA, Cameron NE (1997) Increased oxidative stress in experimental diabetic neuropathy. Diabetologia 40 [Suppl 1]:A549 (Abstract)

42. Keegan A, Cotter MA, Cameron NE (1997) Autonomic neuropathy, corpus cavernosum innervation and endothelial responses: diabetic defects prevented by alpha-lipoic acid in rats. J Peripher Nerv Syst 2: 277 (Abstract)

43. Cameron NE, Cotter MA, Dines KC, Maxfield EK (1993) Pharmacological manipulation of vascular endothelium in non-diabetic and streptozotocin-diabetic rats: effects on nerve conduction, hypoxic resistance and endoneurial capillarization. Diabetologia 36: 516-522
44. Ward KK, Low PA, Schmelzer JD, Zochodne DW (1989) Prostacyclin and noradrenaline in peripheral nerve of chronic experimental diabetes in rats. Brain 112: 197-208

45. Cotter MA, Cameron NE (1997) Effects of dietary supplementation with arachidonic acid rich oils on nerve conduction and blood flow in streptozotocin-diabetic rats. Prostaglandins Leukot Essent Fatty Acids 56: 337-343

46. Cameron NE, Cotter MA, Dines KC, Maxfield EK, Carey F, Mirrlees DJ (1994) Aldose reductase inhibition, nerve perfusion, oxygenation and function in streptozotocin-diabetic rats: dose-response considerations and independence from a myo-inositol mechanism. Diabetologia 37: 651-663

47. Neuringer M, Anderson GJ, Connor WE (1988) The essentiality of $n$ - 3 fatty acids for the development and function of the retina and brain. Annu Rev Nutr 8: 517-541

48. Croset M, Sala A, Folco G, Lagarde M (1988) Inhibition by lipoxygenase products of TXA2-like responses of platelets and vascular smooth muscle: 14-hydroxy from 22: $6 n-3$ is more potent than 12-HETE. Biochem Pharmacol 37: $1275-1280$ 